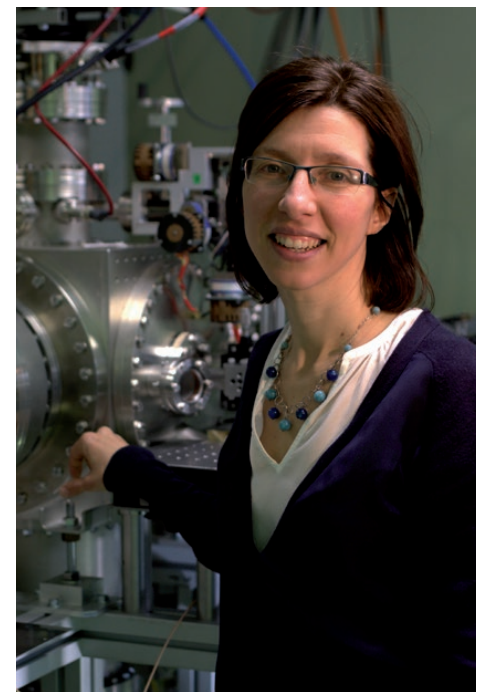

\title{
Angela Vella, sondeuse atomique de la matière
}

Spécialiste de la sonde atomique et chercheuse éclectique, Angela Vella, enseignante-chercheuse au Groupe de physique des matériaux

(CNRS-Université de Rouen), a reçu en 2015 la médaille de bronze du CNRS.

Chaos, cristaux liquides, microcavités laser à base de polymères, sonde atomique... Le spectre des thématiques abordées par Angela Vella au cours de sa carrière est pour le moins étendu. Pour cette jeune physicienne d'origine italienne et tout juste lauréate de la médaille de bronze du CNRS, peu importe la question, l'important " est de comprendre comment ça marche ", confie-t-elle. Puis de "vite se renouveler afin de retrouver l'étincelle de la nouveauté!".

Son parcours est mené tambour battant de Naples à Rouen, en passant par Montpellier et Cachan. Dans le Groupe de physique non linéaire de l'Université de Naples, où elle prépare son doctorat, Angela Vella commence par s'intéresser aux mouvements induits par laser au sein de cristaux liquides. "Il s'agissait de comprendre comment apparaissent parfois des dynamiques chaotiques ", explique-t-elle.

Elle poursuit en 2003 avec un premier stage postdoctoral au Groupe de dynamique des phases condensées, à Montpellier, où la jeune physicienne étudie la stabilisation et la manipulation de défauts dans les cristaux liquides sous l'effet d'un champ électrique. Chose faite en un peu moins d'un an : "Après quelques mois, nous avions compris le phénomène et appris à piloter ces défauts."

Angela Vella opère alors un changement thématique radical : terminés les cristaux liquides. Forte de ses compétences en optique non linéaire, elle opte pour les microlasers à base de polymères dopés par un colorant, à l'École normale supérieure de Cachan. "Il s'agissait de leur donner la bonne forme afin d'obtenir une émission dans une seule direction, avec une bonne accordabilité de la longueur d'onde ", résume-t-elle.

Quelques mois plus tard, la chercheuse est recrutée à l'Université de Rouen sur un poste de maître de conférences. Son nouveau terrain d'investigation : la sonde atomique. Cet instrument unique, dont son laboratoire est leader au niveau mondial, permet de déterminer la structure atomique 3D d'un échantillon, en arrachant puis en analysant un à un les atomes dont il est constitué.

Précisément, début 2005, la technique est alors parfaite pour étudier la structure des métaux en les " attaquant " avec un champ électrique. Mais pour les autres types de matériaux (céramiques, semiconducteurs...), il faudrait pouvoir enrichir la sonde atomique d'un laser femto-seconde, capable d'émettre des impulsions ultra-brèves.

"Les premiers tests avaient été concluants, raconte Angela Vella. Entre modélisation et expérimentation, mon travail a alors consisté à décrire en détails les mécanismes d'éjection des atomes par laser. " Début 2010, l'objectif est atteint pour les métaux. Le travail est toujours en cours pour les autres familles de matériaux. La scientifique ajoute : "Nos idées sur la question sont de plus en plus claires."

Sa volonté d'élargir le spectre des possibilités offertes par la sonde atomique vient sans doute de là. Ainsi, il y a deux ans, Angela Vella a commencé à réaliser une expérience pour collecter la lumière émise par les atomes au moment de leur éjection. "L'idée sous-jacente est de parvenir à coupler les informations fournies par la sonde avec celles obtenues par des mesures de spectroscopie optique et électronique ", détaille la physicienne. En effet, plus récemment, elle a entrepris d'installer un nouveau banc expérimental pour l'analyse des électrons également arrachés aux échantillons de sonde atomique.

"Cela fait parfois un peu peur de s'ouvrir à d'autres choses, il y a toujours le risque que ça ne marche pas", commente Angela Vella. Sa médaille de bronze ne l'y encourage-t-elle pas ? "C'est assurément une reconnaissance, mais qui n'est pas toujours très facile à assumer ", ajoute-t-elle. Cela n'a nullement éteint son désir de comprendre.

Mathieu Grousson, journaliste

\section{- Quelques dates}

\section{Naissance à Atripalda, Italie}

2002 Thèse de doctorat en sciences physiques à I'Université de Naples Federico II

Depuis Maître de conférences à l'Université de Rouen février au sein du Groupe de Physique des Matériaux 2005 (UMR 6634)

2015 Médaille de bronze du CNRS

- Plus d'informations

angela.vella@univ-rouen.fr

Groupe de physique des matériaux (GPM),

Équipe instrumentation scientifique

http://gpm.labos.univ-rouen.fr/spip.php?rubrique48

"Sonde atomique :

la matière comme vous ne I'avez jamais vue »

www.cnrs.fr/inp/spip.php?article2601 